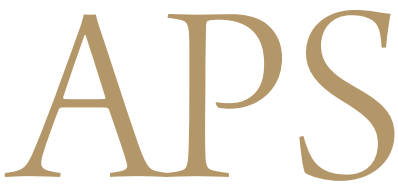

Archives of Plastic Surgery

\title{
Treatment of Pseudoangiomatous Stromal Hyperplasia of the Breast: Implant-Based Reconstruction with a Vascularized Dermal Sling
}

\author{
Bok Ki Jung ${ }^{1}$, Ji Hae Nahm², Dae Hyun Lew ${ }^{1}$, Dong Won Lee ${ }^{1}$ \\ ${ }^{1}$ Institute for Human Tissue Restoration, Department of Plastic and Reconstructive Surgery, ${ }^{2}$ Department of Pathology, Yonsei University \\ College of Medicine, Seoul, Korea
}

\begin{abstract}
Pseudoangiomatous stromal hyperplasia (PASH) of the breast is a benign mesenchymal lesion with incidental histologic findings. Surgical excision is recommended as the treatment of choice for PASH, although the recurrence rates after excision range from $15 \%$ to $22 \%$. A 46-year-old-female presented with a six-month history of bilateral breast enlargement and painful sensation mimicking inflammatory carcinoma. Imaging studies demonstrated innumerable enhancing nodules in both breasts. Due to the growth of the lesions and progressive clinical symptoms, bilateral subcutaneous mastectomy was performed. Grossly, the specimens were round and well-circumscribed, and the histologic examination revealed PASH. After mastectomy, we created a pocket with the pectoralis major muscle and a lower skin flap, which was deepithelized. Anatomical mammary implants were inserted, and the nipple areolar complex was transferred to a new position as a free graft. The aesthetic result was satisfactory after twelve months of follow-up.
\end{abstract}

Keywords Pseudoangiomatous stromal hyperplasia / Breast / Mammaplasty
Correspondence: Dong Won Lee Department of Plastic and Reconstructive Surgery, Yonsei University College of Medicine, 50 Yonsei-ro, Seodaemun-gu, Seoul 03722, Korea

Tel: +82-2-2228-2215

Fax: +82-2-393-6947

E-mail:xyphoss@yuhs.ac

\section{INTRODUCTION}

Pseudoangiomatous stromal hyperplasia (PASH) is a benign proliferative lesion of the breast stroma first described in 1986 by Vuitch et al. [1] PASH can present as clinically mimicking various benign or malignant conditions [2]. Patients with tumorous $\mathrm{PASH}$ generally present a slow-growing breast mass. On physical examination, patients usually present with a solitary, firm, painless, palpable, well-circumscribed, and freely mobile breast mass [3]. On ultrasonography, they commonly demonstrate a solid, well-circumscribed, homogenous, hypoechoic mass, or sometimes, one with ill-defined borders. Definitive diagnosis of PASH requires histopathologic evaluation [4]. Wide local excision is the recommended treatment when there is large tumorous PASH or histologic confirmation is not performed [1]. The decision on the extent of the operation depends on the size of the mass and clinical symptoms. Some cases with diffuse involvement or recurrence may necessitate mastectomy [5].

Some authors have described the clinical relevance, presentation, pathologic features, and treatment of PASH [1-7]. However, to date no data on the reconstructive method of tumorous PASH of the breast after surgical mastectomy have been published. Therefore, we present our surgical treatment using a mammary implant with a vascularized dermal sling for reconstruction after subcutaneous mastectomy of rapidly growing tumorous $\mathrm{PASH}$ of the breast. 


\section{IDEA}

A 46-year-old-female presented with six-month history of progressive bilateral breast enlargement, a painful sensation, upper extremity pain, and back pain. Upon physical examination, giant movable masses with tenderness of the bilateral breast were palpated. Discoloration and thickened skin were noted (Fig. 1). Ultrasonography showed diffuse skin thickening, increased hypoechoic tubular density and edema throughout the parenchyma of both breasts. Magnetic resonance imaging revealed innumerable enhancing nodules in both breasts (Fig. 2). No axillary or internal mammary lymphadenopathy was noted. Differential diagnoses included inflammatory breast cancer, severe mastitis, and phyllodes tumor. The patient reported a family history of breast cancer. Due to the growth of the lesions, progressive clinical symptoms, and family history, a decision was made to perform bilateral subcutaneous mastectomy with immediate reconstruction.

In a standing position, a design for immediate reconstruction was made preoperatively (Fig. 3). A diameter of $4 \mathrm{~cm}$ for the new nipple areolar complex was chosen. Under general endotracheal anesthesia, the patient was laid in a supine position. After subcutaneous total mastectomy, subpectoral dissection was performed

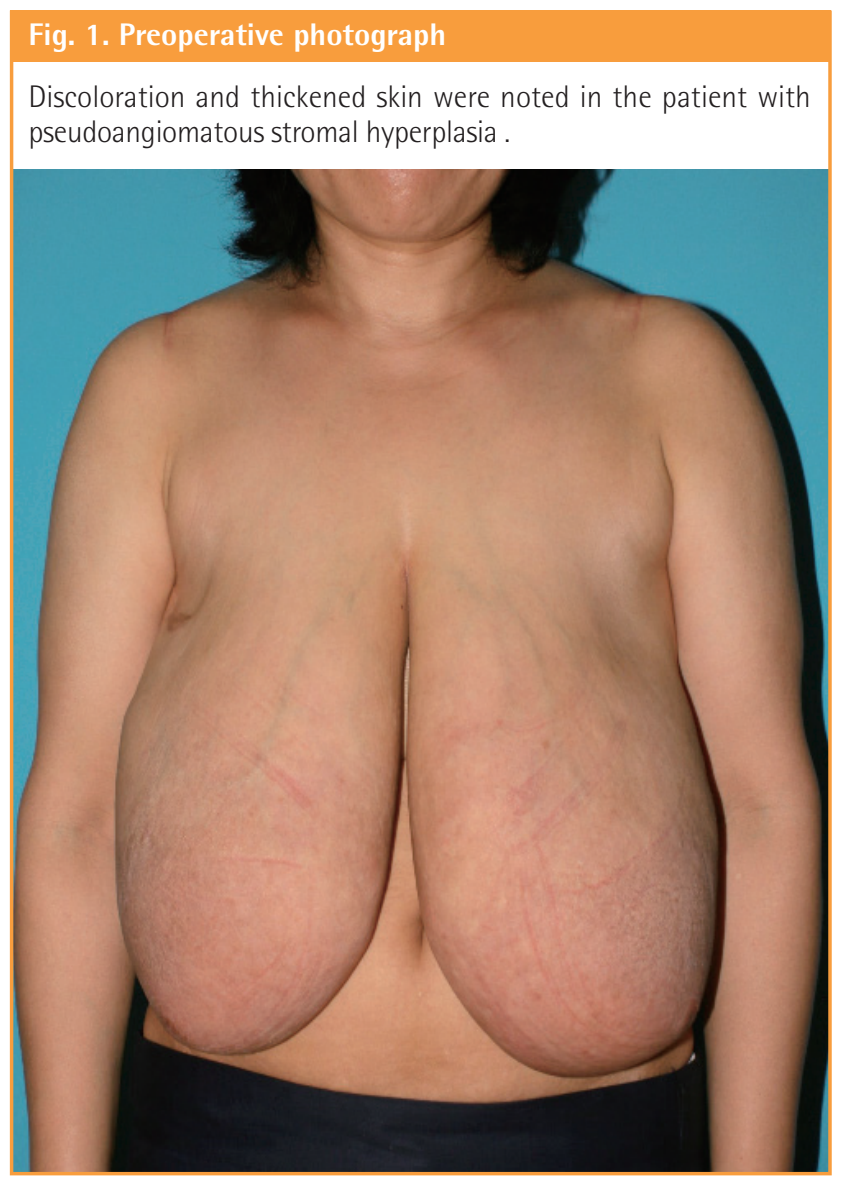

on the lateral border and inferior origin of the pectoralis major muscle with a Bovie electrocoagulator. We constructed a pocket with the pectoralis major muscle and lower skin flap, which was deepithelized like an acellular dermal matrix (ADM) sling applied in breast reconstruction with a mammary implant (Fig. 4).

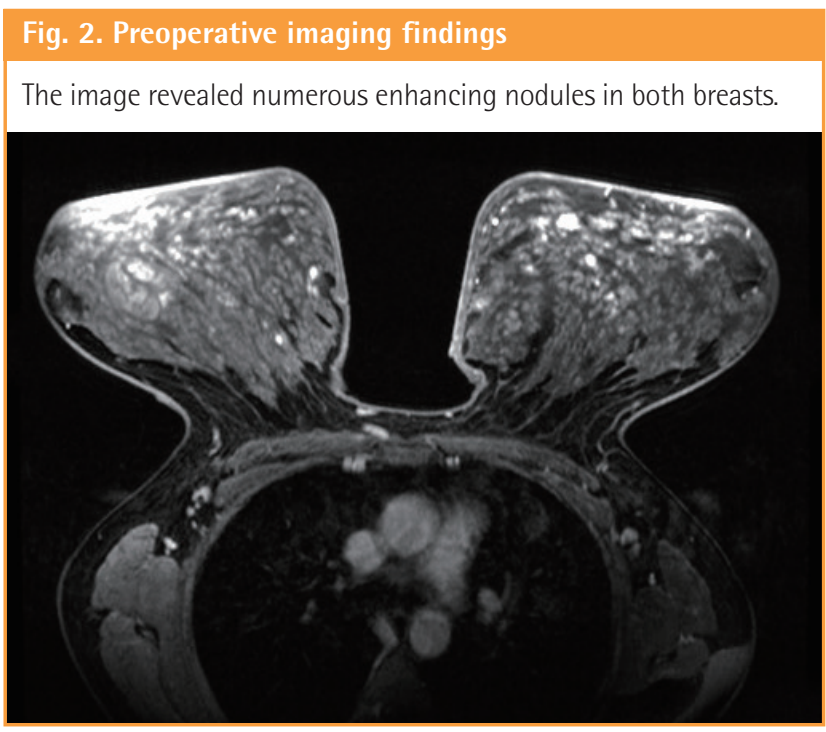

Fig. 3. Operative design

In a standing position, the design was applied to the skin preoperatively. A diameter of $4 \mathrm{~cm}$ was chosen for the new nipple areolar complex; the new distance from the mid-clavicle to nipple was $19 \mathrm{~cm}$ and that from the nipple to the inframammary fold line was $8 \mathrm{~cm}$.

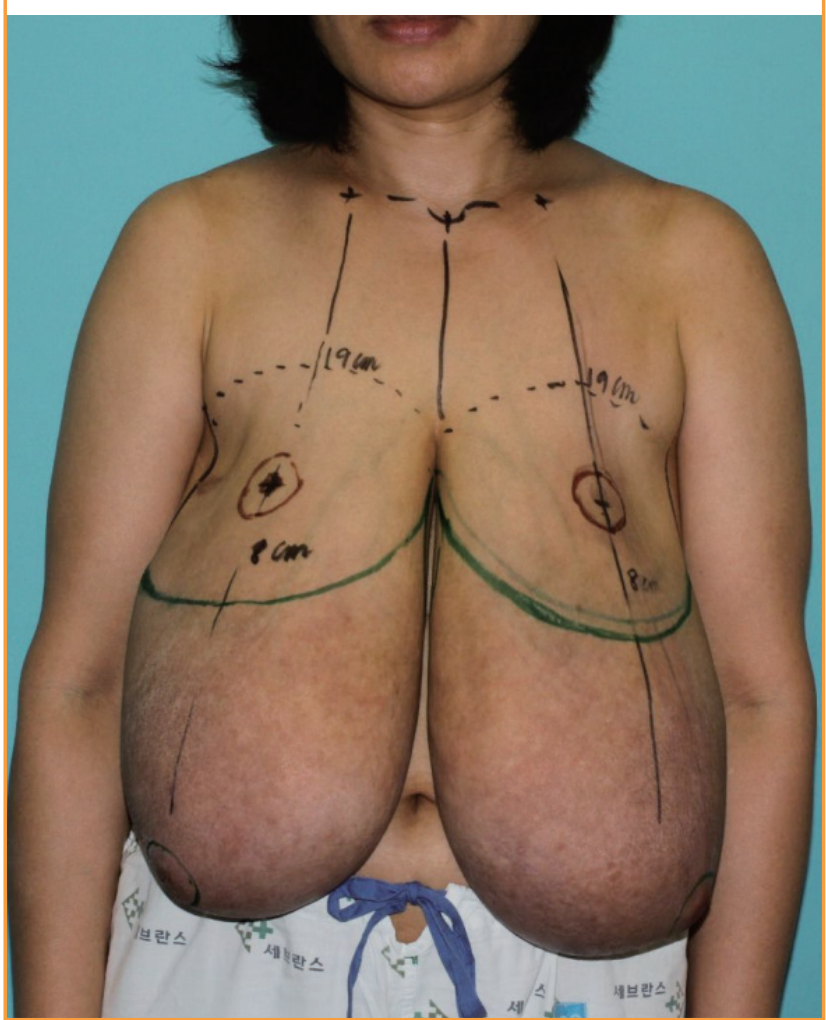


To prevent the formation of hematoma from the bleeding of the deepithelized skin flap, meticulous hemostasis with electric coagulation was performed, and a hemovac drain was inserted beneath the inferior skin flap. Anatomical mammary implants were inserted; then the upper skin flap was draped and fixed to create

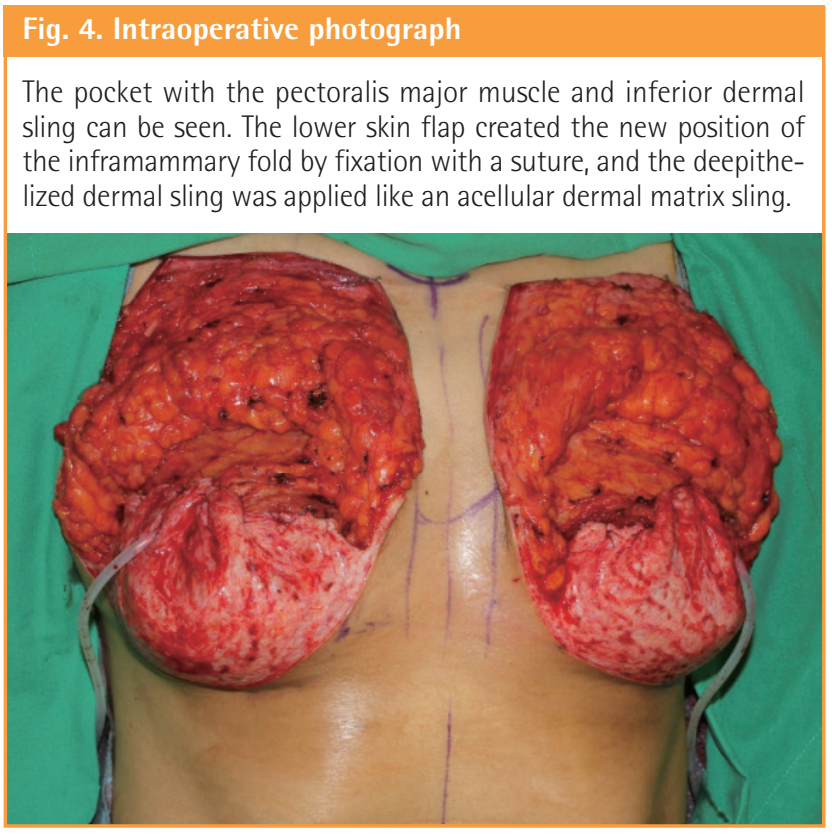

a breast mound with the inframammary fold line created from the lower skin flap. The nipple areolar complex was transferred to a new position as a free graft. The patient had excellent cosmetic results postoperatively (Fig. 5). Grossly, the specimens were round and well-circumscribed, measuring $32 \mathrm{~cm} \times 27 \mathrm{~cm}$ $\times 4 \mathrm{~cm}$ each, with the right breast $3,035 \mathrm{~g}$ and the left $2,832 \mathrm{~g}$

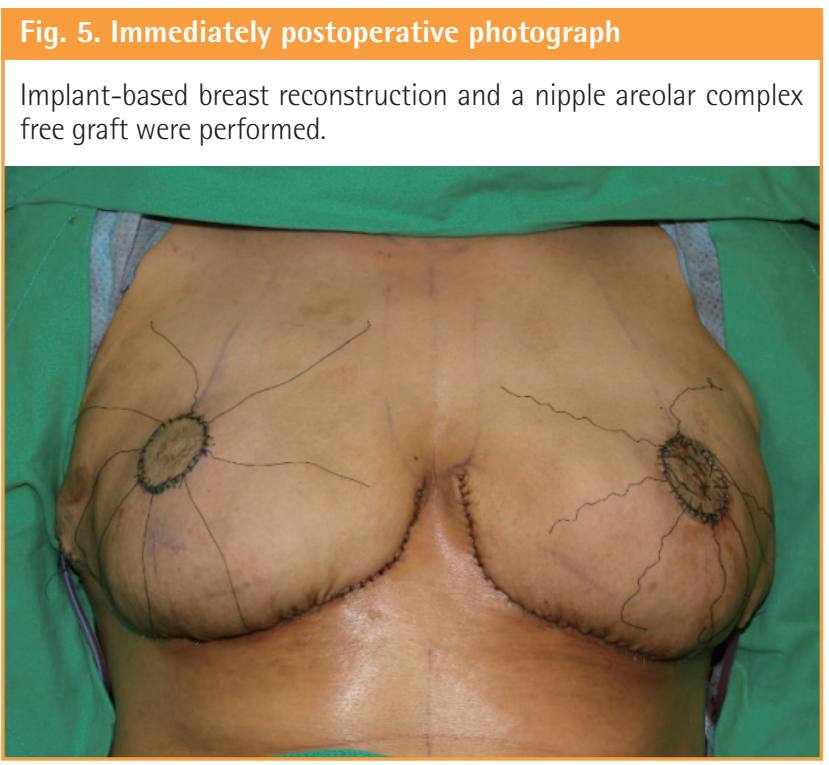

Fig. 6. Gross appearance of the specimen

The specimens were round and well-circumscribed, measuring $32 \mathrm{~cm} \times 27 \mathrm{~cm} \times 4 \mathrm{~cm}$ each. (A) The specimen from the right breast was $3,035 \mathrm{~g}$ and (B) that from the left was $2,832 \mathrm{~g}$.
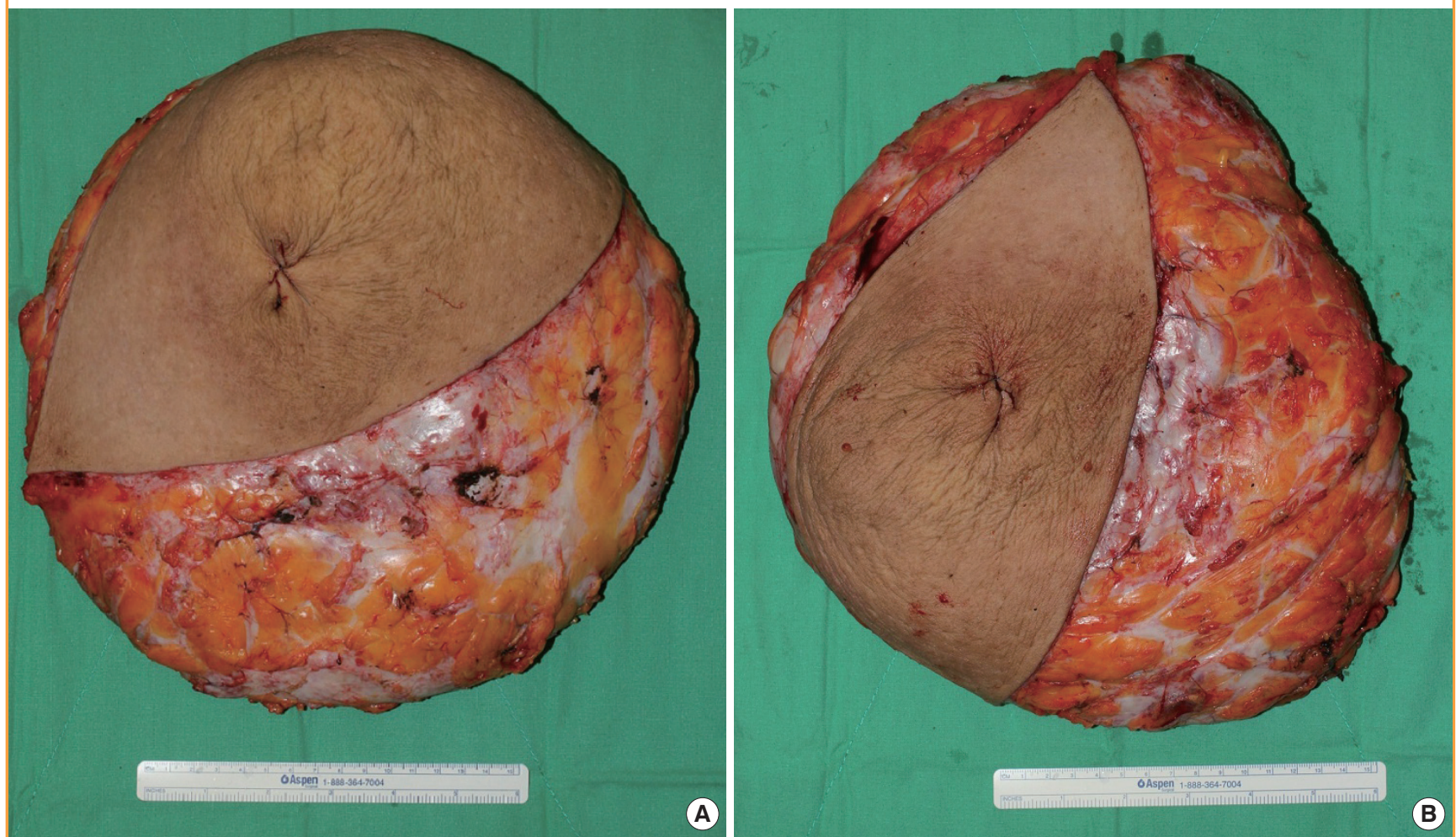


\section{Fig. 7. Histologic examination following bilateral mastectomy}

(A) Low-power view $\left(H \& E_{1} \times 40\right)$ : slit-like, anastomosing spaces in dense collagenous stroma around a lobar structure are seen. (B) High-power view $\left(H \& E_{1} \times 200\right)$ : connected slits outlined by myofibroblasts with uniform, small flat nuclei at the edges of the spaces resemble endothelial cells.

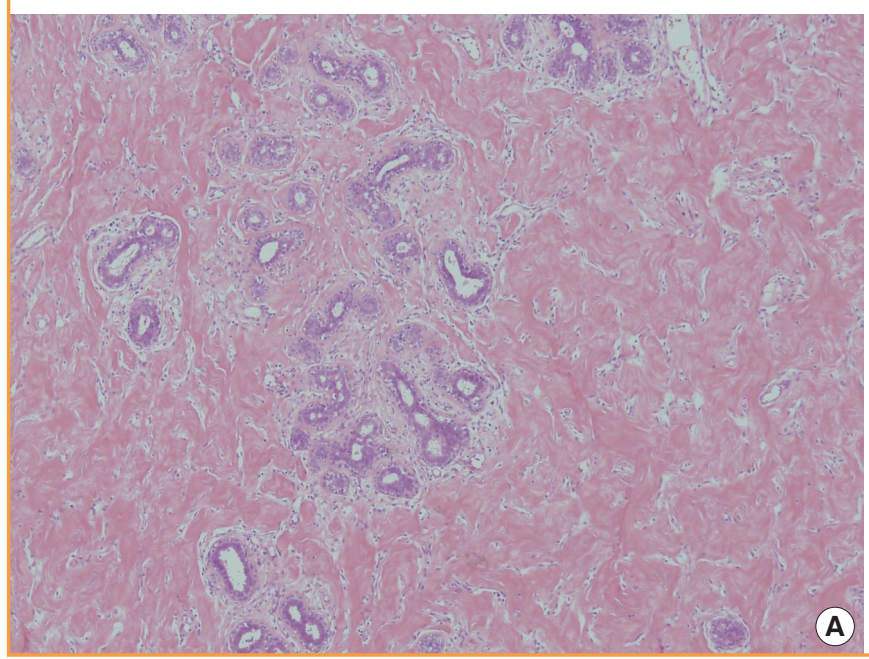

\section{Fig. 8. Outcome at the 12-month follow-up}

Outcome at the 12-month follow-up, without evidence of recurrence.

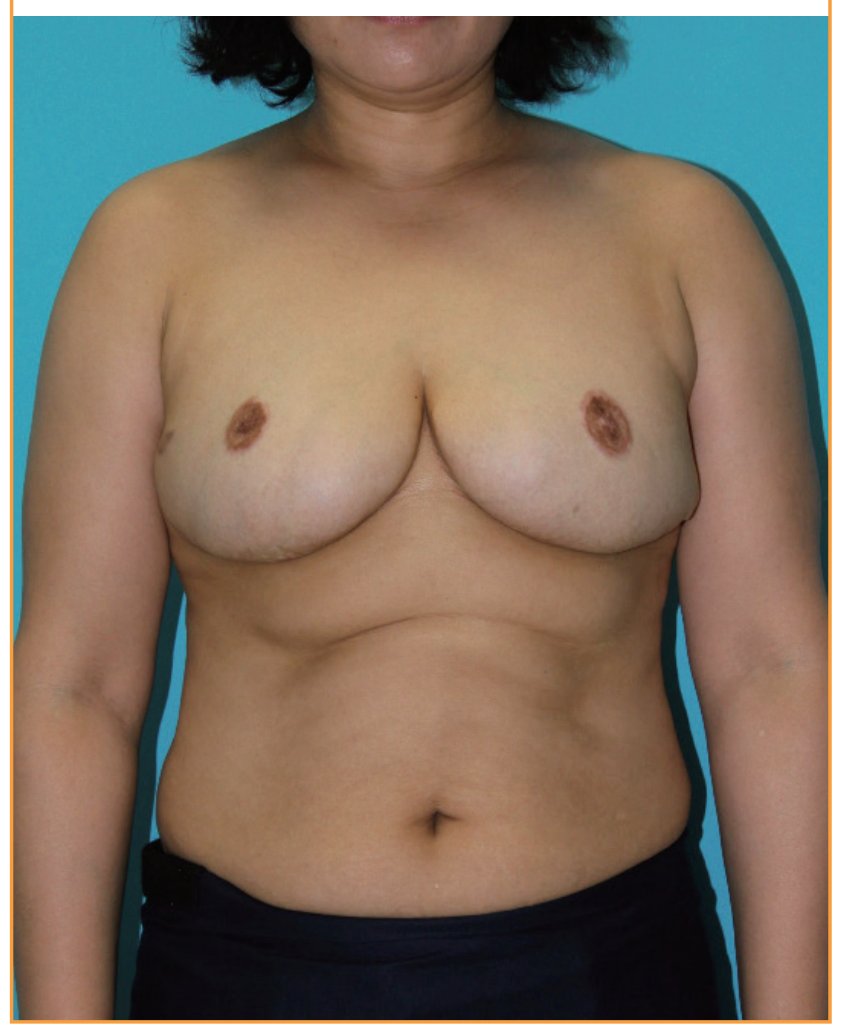

(Fig. 6). The histologic examination revealed characteristic features of diffuse PASH (Fig. 7). The patient underwent followup monitoring for 12 months without evidence of recurrence (Fig. 8).

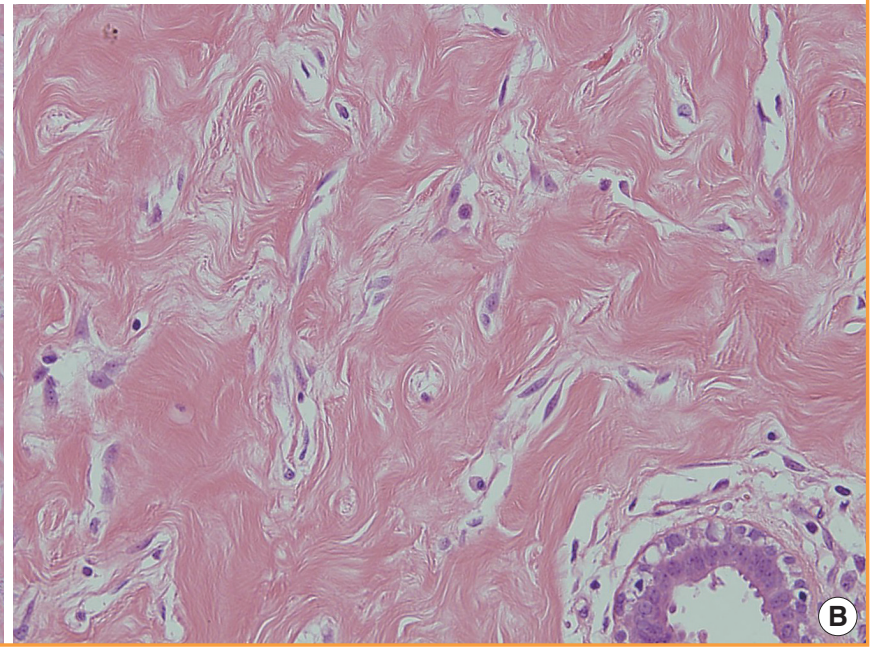

\section{DISCUSSION}

The appropriate treatment for PASH varies. When the lesion is small and asymptomatic, surgical excision is not indicated [4]. If there are suspicious features of malignancy clinically or radiologically, wide local excision is recommended for treatment [6]. For giant tumorous PASH, surgical excision should be performed as the treatment of choice. Some cases with diffuse involvement or multiple recurrences may necessitate mastectomy [7]. Singh et al. [5] noted that after surgical excision, the recurrence rate has been reported to be $15 \%$ to $22 \%$ of cases. The reasons are known to be the incomplete excision of the PASH or development of new lesions.

In our case, as giant tumorous PASH and a family history of breast cancer were noted, bilateral subcutaneous mastectomy was performed. To reduce the risk of recurrence due to remnant tissue, all of the glandular tissue was resected. The defect was reconstructed with anatomical mammary implants.

$\mathrm{ADM}$ is frequently used in implant or expander-based breast reconstruction. A tissue expander or implant is positioned on the subpectoral plane, and the $\mathrm{ADM}$ is used for coverage of the lower pole of the breast. There are many reasons for application of an $\mathrm{ADM}$ sling in implant-based breast reconstruction [8]. First, the ADM sling produces the natural shape of the breast. Second, the sling defines the position of the inframammary fold. Third, the sling reduces the incidence of capsular contracture. However, ADM is associated with significantly increased cost and is also vulnerable to infection [9]. In our case, because the skin envelope of the breast had redundancy and was sufficiently sized, a de-epithelialized flap (dermal sling) replaced the ADM 
for coverage of the lower portion of the implant. The deepithelized dermal sling method compensates for the shortcomings of an $\mathrm{ADM}$ sling; that is, the sling is a more cost-effective technique in patients who are candidates for $\mathrm{ADM}$ in implant-based breast reconstruction. Furthermore, because the implant is covered with a de-epithelialized vascularized dermal sling, the dermal sling is effective against infection.

The pedicle was too long for nipple reconstruction using the inferior or supero-medial pedicled technique; doing so would have endangered the survival of the nipple. Therefore, a full-thickness nipple free graft was performed. The nipple areolar complex was transferred to a new position, which was $8.0 \mathrm{~cm}$ superior to the inframammary fold and $19.0 \mathrm{~cm}$ inferior to the midclavicle. However, since the amount of resected glandular tissue was excessive, the stretched skin was contracted by reducing its weight. As a result, the distance between the mid-clavicle and nipple was underestimated, and postoperatively, the new nipple areolar complex was positioned higher than expected. However, compared to postoperative areolar tattooing, a free nipple graft still produces better cosmetic outcomes, such as a realistic shape and color.

After subcutaneous mastectomy as treatment for tumorous PASH of the breast, the method of implant-based reconstruction using an inferior dermal sling and free nipple graft is reliable and produces excellent cosmetic results, as evidenced by the case we report here.

\section{REFERENCES}

1. Vuitch MF, Rosen PP, Erlandson RA. Pseudoangiomatous hyperplasia of mammary stroma. Hum Pathol 1986;17:18591.

2. Takagi H, Miyairi J, Hata M, et al. A case of tumor-forming pseudoangiomatous stromal hyperplasia of the breast. Breast Cancer 2013;20:187-90.

3. Virk RK, Khan A. Pseudoangiomatous stromal hyperplasia: an overview. Arch Pathol Lab Med 2010;134:1070-4.

4. Salemis NS. Giant tumoural pseudoangiomatous stromal hyperplasia of the breast in the adolescence. ANZ J Surg 2011; 81:469-70.

5. Singh KA, Lewis MM, Runge RL, et al. Pseudoangiomatous stromal hyperplasia: a case for bilateral mastectomy in a 12year-old girl. Breast J 2007;13:603-6.

6. Masannat YA, Whitehead S, Hawley I, et al. Pseudoangiomatous stromal hyperplasia: a case report. Case Rep Med 2010;2010:549643.

7. Powell CM, Cranor ML, Rosen PP. Pseudoangiomatous stromal hyperplasia (PASH): a mammary stromal tumor with myofibroblastic differentiation. Am J Surg Pathol 1995; 19:270-7.

8. Lardi AM, Ho-Asjoe M, Mohanna PN, et al. Immediate breast reconstruction with acellular dermal matrix: factors affecting outcome. J Plast Reconstr Aesthet Surg 2014;67:1098105.

9. Krishnan NM, Chatterjee A, Van Vliet MM, et al. A comparison of acellular dermal matrix to autologous dermal flaps in single-stage, implant-based immediate breast reconstruction: a cost-effectiveness analysis. Plast Reconstr Surg 2013; 131:953-61. 